Research Article

\title{
Analysis of Resistance Characteristics of a 37 Rod Fuel Bundle under Low Reynolds Number
}

\author{
Yonghua Li, Meijun Li (iD, and Yangyang Guo \\ North China Electric Power University, 619 Yonghua North Street Baoding, 071003, China \\ Correspondence should be addressed to Meijun Li; lmj150125@163.com
}

Received 26 July 2020; Revised 17 November 2020; Accepted 26 November 2020; Published 3 December 2020

Academic Editor: Siamak Hoseinzadeh

Copyright (c) 2020 Yonghua Li et al. This is an open access article distributed under the Creative Commons Attribution License, which permits unrestricted use, distribution, and reproduction in any medium, provided the original work is properly cited.

\begin{abstract}
During the working period of decay heat removal system, the flow rate of liquid sodium in wire-wrapped fuel assembly is very low, generally $\mathrm{Re}<1000$. In the present study, both experimental methods and numerical simulation methods are applied. First, water experiment of 37-pin wire-wrapped rod bundle was carried out. Then, the numerical simulation study was carried out, the experimental data and the numerical simulation results were compared and analyzed, and a suitable turbulence model was selected to simulate the liquid sodium medium. Finally, numerical simulations under different boundary conditions were performed. Results indicate that except for the low Reynolds number $k$ - $\varepsilon$ turbulence model, other turbulence models have little difference with the experimental results. The results of realizable $k-\varepsilon$ turbulence model are the most close to the experimental results. Compared with the friction factor obtained by using water medium and liquid sodium medium, the calculation results of water medium and sodium medium under the same condition are basically consistent, with the deviation within $1 \%$. The reason is that the velocity of water is higher than sodium medium at the same Reynolds number, and the transverse disturbance caused by helical wire is larger.
\end{abstract}

\section{Introduction}

Nuclear power, which has been around for decades, is one of the essential powers in energy fields. Belonging to it, nuclear power generation is at a rough estimate accounting for $16 \%$ of the whole world power systems. As one of the fourthgeneration nuclear reactors, the sodium-cooled fast reactor (SFR) can best meet the types of commercial nuclear power plants. SFR can utilize uranium more efficiently and burn up long-lived actinides. The passive safety technology is generally used in a sodium cooled fast reactor, which is introduced into the third-generation nuclear power plant. The passive residual heat removal system uses the natural circulation generated by the density difference under the action of fluid gravity to take out the heat from the core during the accident condition. The natural circulation in the core can be divided into two ways: the interwrapper flow and the inner subassemblies flow. The friction resistance of the inner sub- assemblies accounts for about $90 \%$ of the total circulation loop. Therefore, the key of condensate flow rate and the heat taken out under decay heat removal conditions is the thermal-hydraulic behavior of fuel assembly.

Liu and $\mathrm{Yu}[1]$ proposed the ICRT pressure drop relationship basing on the CRT model and the work of Engel et al. 's research improves SUPERENERGY code and verifies them by fluid mechanics CFX. Gajapathy et al. [2] make the analysis of the impact on the helical wires around a 217-pin fuel bundle at high Reynolds number equal to 95000 by computational fluid dynamics (CFD), the research of the resistance friction tending to be reduced as the helical pitch becoming increased, and the conclusion of the temperature gradient getting down with the transverse flowing in the assembly. Lyu et al. [3] make the study of the resistance characteristics of 61-wire-wrapped fuel assembly in LeadBismuth eutectic (LBE); the conclusion that the friction factor results in water is obviously bigger than in LBE in that 
the secondary flow induced by helical wire in water is much stronger than in LBE at the same Reynold number. Kamide et al. [4] analyzed inter-subassembly heat transfer during natural circulation decay heat removal in fast breeder reactors. The result show inter-subassembly heat transfer will significantly influence the flow and temperature distributions in subassemblies. Sarafraz et al. [5, 6] studied the flow characteristics of liquid metal in a microchannels. Hoseinzadeh et al.'s [7] study is to investigate the pulsating flow in a three-dimensional channel. Channel flow is laminar and turbulent. After validation, the effect of different channel crosssectional geometries (circular, hexagonal and triangular) with the pulsating flow is investigated. Chun and Seo [8] evaluated the different existing friction correlations by comparing 14 groups of published experimental data. Their results indicate that detailed Cheng and Todreas (CTD) correlations give the best agreement with experimental data for all flow regions. Bubelis and Schikorr [9] also selected 21 sets of experimental data for evaluation. They concluded that the Rehme (REH) correlation performs the best. Cheng and Todreas [10] presented a series of experiments on the 37pin rod bundle test section. He used isokinetic sampling probes to measure the flow rate of the subchannels and evaluated the mixing by introducing a salt tracer into the bundle. Chang et al. [11, 12] also adopted the isokinetic sampling method to collect the flow at subchannels. And new sampling probes have been devised. Seyyed [13] et al. present a hybrid finite-element/finite-difference approach. The implemented hybrid scheme requires less computation time compared with the conventional 3-D finite-element method, requiring less program coding. Cheng et al. [14] analyzed the hydraulic characteristics of different kinds of wire-wrapped rod bundle assemblies of China Experimental Fast Reactor (CEFR) under low Reynolds number by using CFX program. The results show that the calculation result of hydraulic characteristic under low Reynolds number is in good agreement with the Engel's relational expression.

Liquid sodium flowing in wire-wrapped fuel assembly is considered low during the decay heat removal, generally $\mathrm{Re}$ $<1000$. Now, there come few materials referable on resistance characteristic at low Reynolds number. In this article, we have tested the resistance in the 37-rod assembly at low Reynold number, with water acting as a medium. We also used fluid mechanics CFD in calculation. We have determined the relatively agreeable model, turbulence model after many tests. Plus, we have done the commutative analysis on the flow under the heating, with sodium acting as a medium. The overall study was made to illustrate the impact on resistance characteristics in the 37-rod fuel assembly under the heating.

\section{Water Test Section}

2.1. Structural Parameters for Fuel Assembly. The parameters in the experiment are referred from the Liquid Metal Technology Laboratory of Tsinghua University, in which the design specifications are listed in Table 1. The fuel bundle is gathered in a hexagonal tube with a $55 \mathrm{~mm}$ flat-to-flat distance inside. The 37 pins ( $7 \mathrm{~mm}$ in diameter) is wrapped by
TABLE 1: Geometric parameters of test section.

\begin{tabular}{lc}
\hline Geometric parameters & Values \\
\hline Number of pins & 37 \\
Pin diameter & $7 \mathrm{~mm}$ \\
Pin pitch & $8.6 \mathrm{~mm}$ \\
Pin axial length & $2700 \mathrm{~mm}$ \\
Heated length & $1000 \mathrm{~mm}$ \\
Heat flux distribution & Uniform \\
Tube flat-to-flat distance & $55 \mathrm{~mm}$ \\
Wire spacer diameter & $1.5 \mathrm{~mm}$ \\
Wire lead pitch & $150 \mathrm{~mm}$ \\
\hline
\end{tabular}

wire spacers ( $1.5 \mathrm{~mm}$ in diameter) and covered with a wrapping lead of $150 \mathrm{~mm}$. Electrically heated length of the fuel rods is $1000 \mathrm{~mm}$ whose position is at $1200 \mathrm{~mm}$ downstream of the fuel assembly bottom.

2.2. Experimental Device. The experiment is under room temperature and pressure, with water acting as a medium. Experimental equipment includes stabilized water tank, flow control valve, measuring elements, container for weight, water flow collector, mass measuring device, temperature measuring device, and rubber hose etc. The device design drawing is shown in Figure 1. The stabilized water tank is for the purpose of keeping the inlet pressure constant. Flow control valve is in flow adjustment from 0 to $15 \mathrm{lpm}$. A tee is installed at the inlet of the test assembly which connects a stabilized water tank and glass tube. The tube with a scale can measure the inlet pressure. The measuring set is oblique at a certain angle, and the outlet flow level remains the same in the measuring process. The water flow collector is equipped at the outlet to collect the water and measure its flowing mass over a certain period of time.

2.3. Measurement Deviation Analysis and Correction. In the experiment, keeping the water level in the tank smooth means the pressure inside is unchanged. The regulating valve is designed to regulate the flow. Measurement has been made when the flow is in the level after each regulating in order to minimize the deviation. The resistance value is determined by the level difference between the vertical glass tubes and the outlet, whose maximum deviation is $9.8 \mathrm{~Pa}$. Mass flow has been gotten from flow measurement from water mass, then converted into volume flow with the measurement accuracy of about $0.15 \mathrm{lpm}$. Flow and pressure measurements are conducted at the same time; meanwhile, the water temperature in the assembly and in the glass tubes is recorded. The water temperature basically remains unchanged. However, in the glass tubes, the water temperature is easily influenced by room temperature if the water is still. And the temperature difference leads to the difference of flow density between the glass tube and the assembly resulting in measurement deviate.

Except for the measurement deviation, we also need to make corrections on the local resistance coefficients in the 


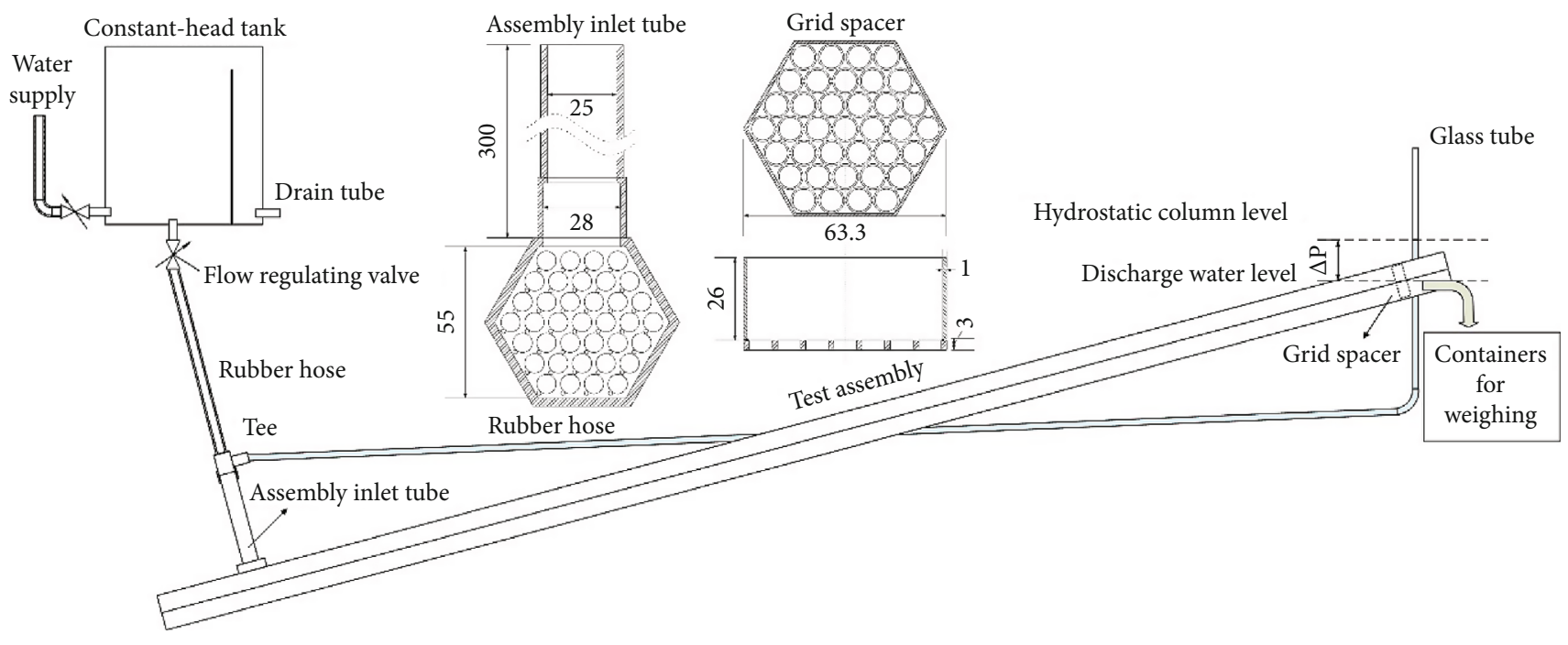

FIgURE 1: Experimental model.

inlet and the outlet. Then, we could calculate the resistance coefficient following the formula below:

$$
\begin{aligned}
\Delta P_{t} & =\left(\rho_{m}-\rho_{b}\right) g l+\rho_{m} g \Delta h, \\
\Delta P_{\mathrm{en}} & =\frac{\left(\lambda\left(L_{\mathrm{en}} / d_{\mathrm{en}}\right)+\varepsilon_{\mathrm{en}}\right)\left(\rho_{\mathrm{en}} v_{\mathrm{en}}^{2}\right)}{2}, \\
\Delta P_{\mathrm{sp}} & =\frac{\left(0.5\left(1-R_{A}\right)+\left(1-R_{A}\right)^{2}\right)\left(\rho_{b} v_{\mathrm{sp}}^{2}\right)}{2}, \\
\Delta P_{t} & =\Delta P_{f}+\Delta P_{\mathrm{en}}+\Delta P_{\mathrm{sp}}, \\
f & =\Delta P_{f} \frac{D_{e}}{L} \frac{2 \rho_{b}(3600 * S)^{2}}{G^{2}} .
\end{aligned}
$$

\section{Numerical Analysis Section}

3.1. Computational Grids. The actual length of the 37 -fuel-rod assembly is $2700 \mathrm{~mm}$ making complex computations, so we take $900 \mathrm{~mm}$ length to simplify the calculation process. The spiral wrapping wire in the assembly not only positions the fuel rods but also stirs the coolant. And the wire makes the inner structure of the assembly more complicated. Practically, the wire and rods are tangential with line contact, as we assume them as tangents. Angles formed by two tangents are zero. In calculation, the geometric topology is theoretically applied to it. However, in the real modeling, there exists no grid element with a zero angle, so we have to deal with surface contact. Gajapathy et al. [2,15-17] regard the structure of all the wires as hexagon in calculation and achieve sound results. Nowadays, it is a conventional way that wires are slightly inserted into the fuel rods without changing the wire structure. Research by Gajapathy [18] show that, although taking the embedded way loss some computing resources, this has no obvious impact on the flow and highly represents the physical model. So, we choose the embedding method. In this stimulation, we slightly inserted the helical wire into the fuel assembly, making the surface contact instead of point contact with $0.05 \mathrm{~mm}$ embedded size and 1/30 of the diameter of the wire.

The helical wire in the assembly causes the internal structure of fuel assembly complex. There appear tiny slits among rods and the $0.1 \mathrm{~mm}$ shortest distance between the wire and the adjacent rods. This kind of structure has a disadvantage in structure meshing. Additionally, that wire on each rod makes the whole structure neither symmetrical nor asymmetrical which makes it hard to do the structure meshing. We introduced a nonstructural mesh which is combined with hexahedral and tetrahedral to ensure the meshing adaptable and the computing efficient. During the encryption, the pressure loss varies as the changing number of grids illustrated in the below Figure 2. The pressure loss reaches $7 \mathrm{~Pa}$ around at the number of grids up to 43 million in which the variation range is less than $1 \%$. In this case, we consider the mesh experimentally workable. For more accuracy and less cost in computing resources, we take 43 million mesh in calculation.

3.2. Boundary Condition. These stimulations are separately conducted with water medium and liquid sodium medium. With water as a medium, the boundary conditions are consistent in the fuel assembly with water experiment. The inlet is the velocity inlet formula according to Reynold value in experiment $\operatorname{Re}=\rho \bar{V} D$. The outlet is as pressure outlet which is regarded air pressure. In this stimulation, under no heating condition, the water physical parameter is a constant with the density of $998.2 \mathrm{~kg} / \mathrm{m}^{3}$ and dynamic viscosity is $1.0003 \times 10^{-3}$.

With liquid sodium as a medium, calculations have been made under heating and nonheating condition, comparison of the results, and analysis of the influence of the resistance in the assembly under heating. The heating condition refers that the heat released from the fuel rods in the assembly when liquid sodium is flowing through the heating section during the process of decay heat removal. The take-out heat from interwrapper flow is far less than fuel-rod generating heat under regular reactor working condition, while the take-out heat from interwrapper reaches up to $30 \%$ to $50 \%$ under accidental decay heat removal. We set up heat transfer boundary to the rod-bundle wall in 


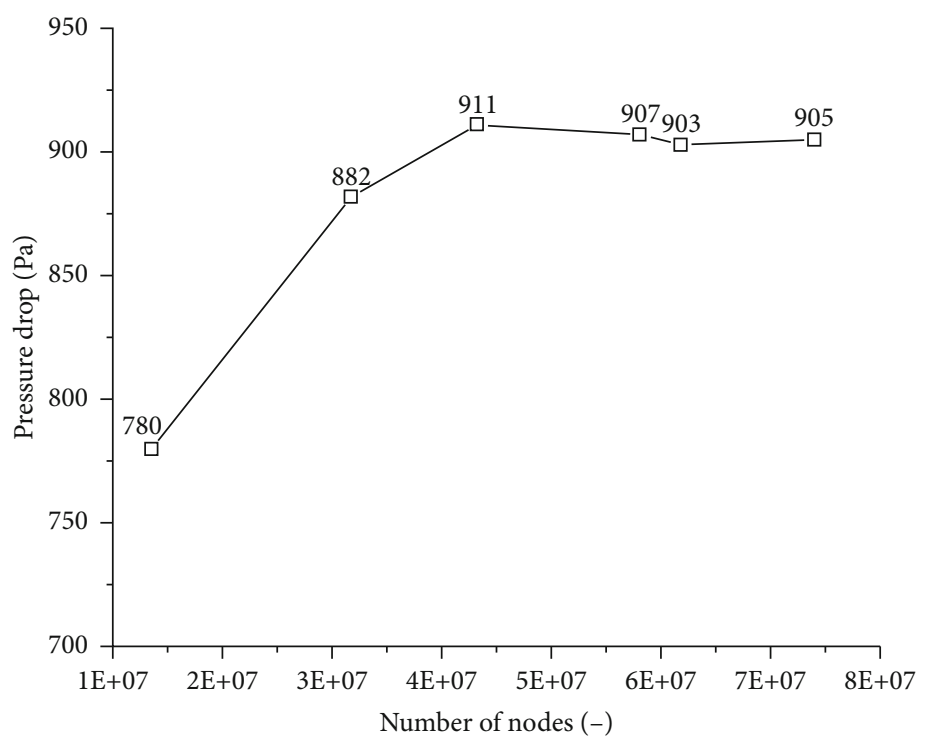

Figure 2: Mesh sensitivity analysis.

which the heat flux is $11 \mathrm{~kW} / \mathrm{m}^{2}$, and the entire heating power accounts for $1.5 \%$ of the actual power in the assembly. The existence of interwrapper flow can effectively reduce the peak temperature of the core during the decay heat removal period [19]. The assembly wall is with heat transfer boundary as well, in which the outward power is in the $40 \%$ in the assembly. Inlet and outlet boundary set-ups are in the same way as water medium. In calculating, the density and dynamic viscosity of sodium has a great effect on the flow, so we set these two parameters as functions changeable with temperature. The parameter settings under different conditions are shown in Table 2.

The viscosity of liquid metal sodium is similar to that of water, so it has very good fluidity. The neutron absorption cross-section of sodium is small, and its moderating ability is weak. Under normal temperature and pressure, the melting point of sodium is $97.82^{\circ} \mathrm{C}$. The melting point is very low, and the boiling point is very high. It can operate continuously under normal pressure and high temperature. So, it is a very good coolant for sodium cooled fast reactor. We calculated the physical properties of sodium according to the following formula [20]:

$$
\rho=\rho_{c}+f\left(1-\frac{T}{T_{c}}\right)+g\left(1-\frac{T}{T_{c}}\right)^{h}
$$

for $371 K \leq T \leq 2503.7$, where $\rho_{c}=219.0, f=275.32, g=$ 511.58, $h=0.5, T_{c}=2503.7$, and $\rho_{c}$ and $T_{c}$ are, respectively, the critical density and critical temperature.

$$
\begin{aligned}
& \lambda=124.67-0.11381 \mathrm{~T}+5.5226 \times 10^{-5} T^{2}-1.1842 \times 10^{-8} T^{3}, \\
& c_{p}=1.6582-8.4790 \times 10^{-4} T+4.4541 \times 10^{-7} T^{2}-2992.6 T^{2} \\
& \eta=\exp (-6.4406-0.3958 \ln (T)+(556.835 / T)
\end{aligned}
$$

\begin{tabular}{|c|c|c|c|c|}
\hline \multirow{2}{*}{ Conditions } & \multicolumn{2}{|c|}{$\begin{array}{l}\text { Heat flux } \\
\left(\mathrm{kW} / \mathrm{m}^{3}\right)\end{array}$} & \multirow{2}{*}{ Medium } & \multirow{2}{*}{$\begin{array}{c}\text { Inlet } \\
\text { temperature }\end{array}$} \\
\hline & $\begin{array}{c}\text { Rod } \\
\text { bundle }\end{array}$ & Wall & & \\
\hline Case 1 & 0 & 0 & \multirow{7}{*}{$\begin{array}{l}\text { Liquid sodium } \\
\text { metal }\end{array}$} & $300 \mathrm{~K}$ \\
\hline Case 2 & 0 & 0 & & \multirow{6}{*}{$573.15 \mathrm{~K}$} \\
\hline Case 3 & 10.9 & -23.4 & & \\
\hline Case 4 & 13.7 & -23.4 & & \\
\hline Case 5 & 8.7 & -23.4 & & \\
\hline Case 6 & 11 & -18.2 & & \\
\hline Case 7 & 11 & -13.7 & & \\
\hline
\end{tabular}

TABLE 2: The parameter settings under different conditions.

\section{Results and Analysis}

4.1. Experimental Calculation Results. After testing, we have the number of pressure loss and mass flow on several water flows in the assembly process and correct the data according to the above formula, then determine the resistance coefficient at some Reynolds number shown in Figure 3. It can be seen that the measurement uncertainty increases with the decrease of Reynolds number. We conclude that the uncertainty in the measuring process becomes increased along with the decrease in low Reynolds number, which makes the deviation larger in measuring and causes the uncertainty increased.

\subsection{Numerical Simulation Results}

4.2.1. Water. Although there are many ways of CFD calculation, the method at low Reynolds number is relatively few. Whether selection of turbulence mode can work as a medium, we stimulated the resistance measuring experiment by the realizable $k-\varepsilon$ model, RNG $k-\varepsilon$ model, low Reynolds number $k-\varepsilon$ model, shear stress transfer $k-\varepsilon$ model, SST model, and laminar flow model comparing experimental 


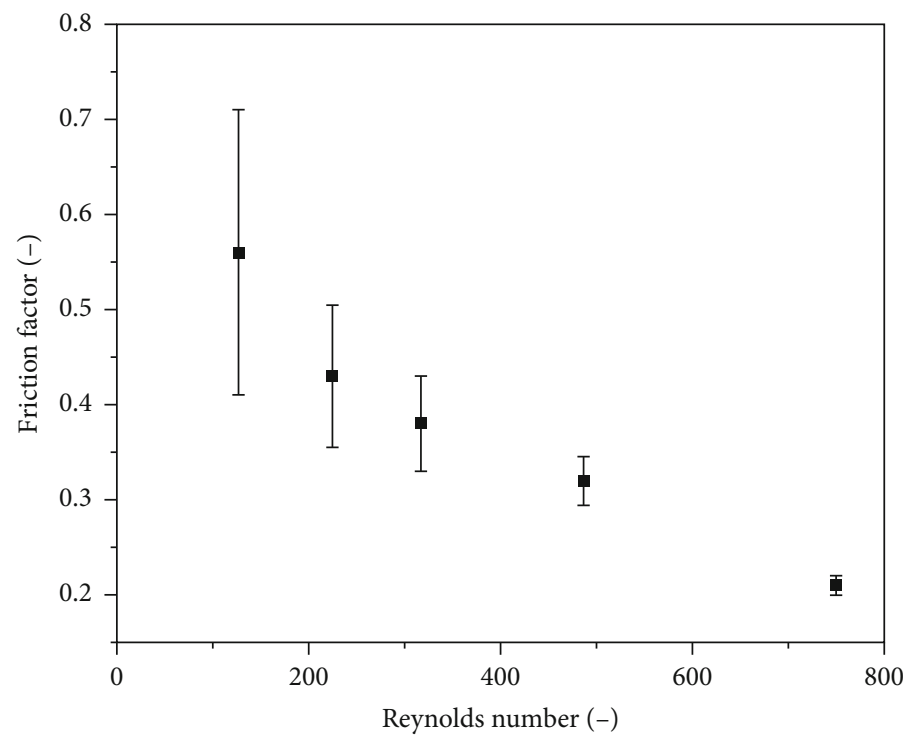

FIgURE 3: Experimental results.

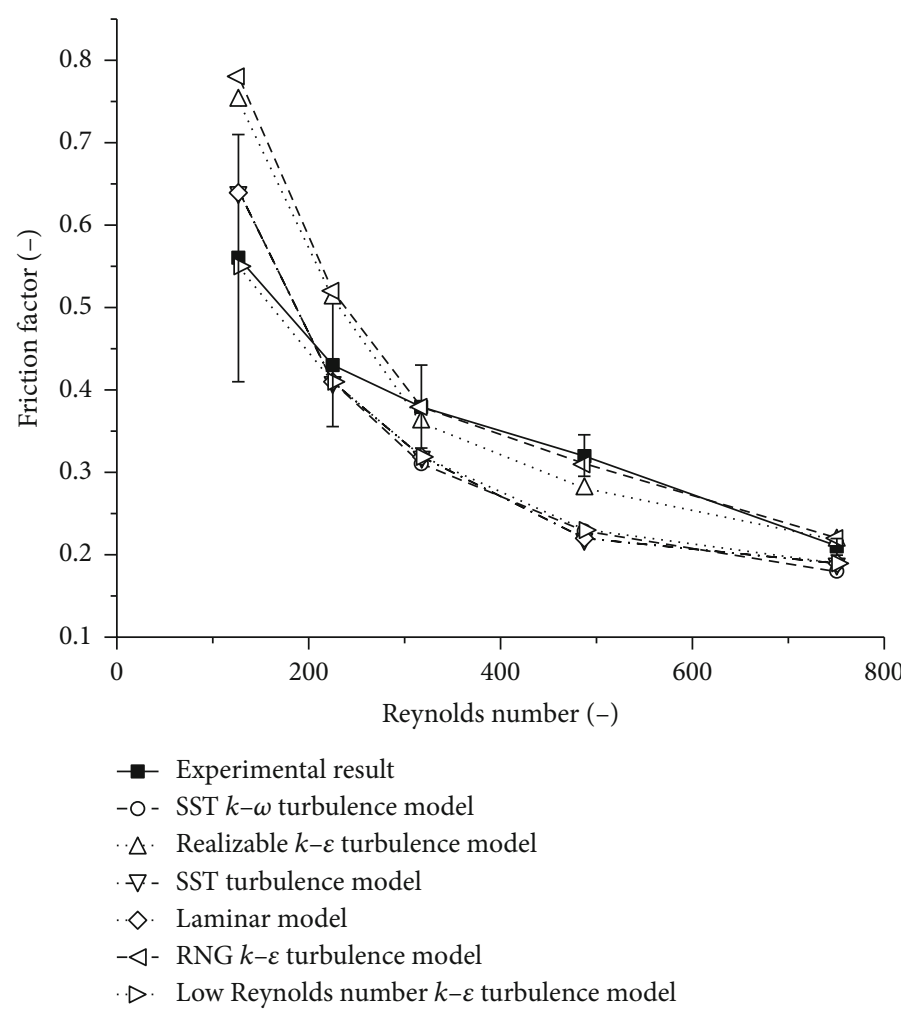

Figure 4: Calculation results of turbulence model.

results with calculations listed in Figure 4. It is found that the average absolute deviation between the calculated results and the experimental results is about $15 \%$. It can be clearly seen that except for the low Reynolds number $k-\varepsilon$ turbulence model when the Reynolds number is less than 100 , the calculation results are quite different from the experimental results, and the calculation results of other models are not much different from the experimental results. The calculation results of the SST $-k-\varepsilon$ model, SST model, and laminar flow model are similar, and there is little difference with the experimental results when the Reynolds number is small. The results of realizable $k-\varepsilon$ model and low Reynolds number $k-\varepsilon$ model are better than other turbulence models. Compared with low Reynolds number $k-\varepsilon$ model, the realizable $k-\varepsilon$ model is in good agreement with experimental data at high Reynolds number. Considering the limitation of computing resources, the realizable $k-\varepsilon$ model is used in the subsequent simulation. 


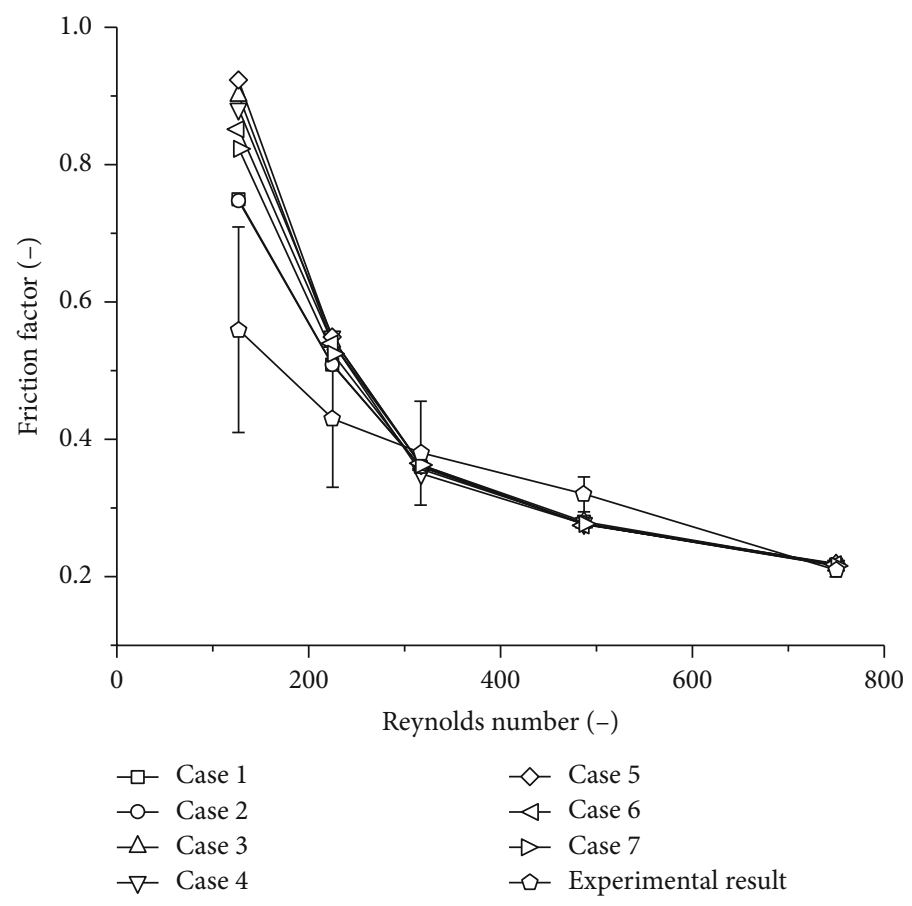

Figure 5: Calculation results of different conditions.

4.2.2. Liquid Metal Sodium. In this paper, the numerical simulation of different conditions is carried out, and the friction factors are shown in Figure 5. It can be seen that the friction factors obtained by Case 3-Case 7 are obviously larger than that by Case 2. These are enough to show that the heating conditions will affect the friction factors of the wirewrapper fuel assembly at low Reynolds number. As the Reynolds number decreases, the drag coefficient of the fuel assembly increases. As the Reynolds number decreases, the increment in the resistance coefficient of each heating condition relative to the constant temperature condition gradually increases. Case 5 has the largest increment of $1.4 \%-24.1 \%$, and Case 7 has the smallest increment of 1.2\%-11.2\%.

It can be seen from the Figure 5 that the calculation results of water medium and sodium medium under the same condition are basically consistent, with the deviation within $1 \%$. According to the Reynolds similarity criterion, the friction factor obtained under the same Reynolds number should be the same. The heating power is changed at Case 3Case 5. It can be seen from Figure 5 that the friction factor calculated by Case 4 is the smallest and the result obtained by Case 5 is the largest. With the same cooling power, the friction factor decreases with the increase of heating power. Comparing the results of Case 3, Case 6, and Case 7, it is found that the friction factor decreases with the decrease of heat sink power.

\section{Conclusion}

Based on a 37-pin wire-wrapped rod bundle, the design of water experiment and computational fluid dynamics (CFD) software FLUENT were designed to conduct calculation for it. The resistance characteristics of 37-pin wire-wrapped rod bundle at low Reynolds number are analyzed; the following conclusions can be drawn:

(1) Compared with the results of CFD and experiment, it can be found that except for the low Reynolds number $k-\varepsilon$ turbulence model, other turbulence models have little difference with the experimental results. The results of realizable $k-\varepsilon$ turbulence model are the closest to the experimental results

(2) Taking liquid sodium as medium, we make calculations under heating and no heating working condition with a reference of Reynold similarity criterion. Comparing the resistance coefficients in water flow and in sodium flow, the coefficients show that the sodium is $1 \%$ less than water under no heating condition because the sodium is flowing slow than water same Reynolds number

(3) The resistance is much larger in heating than in no heating, in which the difference becomes larger at the decreasing in Reynold number. The main reason is that, under heating work condition, the buoyancy causes the flowing redistributed

\section{Nomenclature}

$d_{\text {en }}$ : Inner diameter of inlet pipe $(\mathrm{mm})$

$D_{e}$ : Component length $(\mathrm{mm})$

$f: \quad$ Friction coefficient of assembly (-)

$g: \quad$ Gravitational acceleration $\left(\mathrm{m} / \mathrm{s}^{2}\right)$

G: $\quad$ Mass flow through the assembly $(\mathrm{kg} / \mathrm{s})$

$\Delta h: \quad$ The height difference between the water column level in the glass tube and the liquid level of test assembly $(\mathrm{mm})$ 
$l: \quad$ The height from the inlet pipe of test assembly to the outlet of assembly $(\mathrm{mm})$

$L_{\text {en }}: \quad$ Length of inlet pipe $(\mathrm{mm})$

$\Delta P_{f}:$ The frictional resistance of test assembly against water flow $(\mathrm{Pa})$

$\Delta P_{t}: \quad$ Pressure drop at inlet and outlet of test assembly $(\mathrm{Pa})$

$\Delta P_{\text {en }}$ : Resistance pressure drop of inlet pipe of test assembly $(\mathrm{Pa})$

$\Delta P_{\mathrm{sp}}$ : Local resistance produced by the outlet bracket of test assembly $(\mathrm{Pa})$

$R_{A}$ : Ratio of projected area of outlet bracket on flow section in assembly (-)

$S: \quad$ Flow area in test assembly $\left(\mathrm{m}^{2}\right)$

\section{Greek Symbols}

$\rho_{m}$ : Density of water in vertical glass $\left(\mathrm{kg} / \mathrm{m}^{3}\right)$

$\rho_{b}$ : Density of water flow in the assembly $\left(\mathrm{kg} / \mathrm{m}^{3}\right)$

$\lambda: \quad$ Friction coefficient of inlet pipe (-)

$v_{\text {en }}$ : Velocity in inlet pipe $(\mathrm{m} / \mathrm{s})$

$v_{\mathrm{sp}}$ : The rate of water flow in the assembly through the outlet bracket $(\mathrm{m} / \mathrm{s})$

\section{Data Availability}

It is found that the average absolute deviation between the calculated results and the experimental results is about $15 \%$. The results of realizable $k-\varepsilon$ model and low Reynolds number $k-\varepsilon$ model are better than those of the low Reynolds number $k$ $-\varepsilon$ model. Compared with the low Reynolds number $k-\varepsilon$ model, the realizable $k-\varepsilon$ model is in good agreement with experimental data at high Reynolds number. Considering the limitation of computing resources, the realizable $k-\varepsilon$ model is used in the subsequent simulation. It can be seen that the friction factors obtained by Case 3-Case 7 is obviously larger than that of Case 2. These are enough to show that the heating conditions will affect the friction factors of the wire-wrapper fuel assembly at low Reynolds number. The friction factor decreases with the increase of heating power. Comparing the results of Case 3 and Case 6, it is found that the friction factor decreases with the decrease of heat sink power.

\section{Conflicts of Interest}

The authors declare that they have no conflicts of interest.

\section{References}

[1] L. Yizhe and Y. Hong, "Thermal-hydraulic analysis of fuel subassem blies for sodium-cooled fast reactor," Atomic Energy Science and Technology, vol. 2, pp. 128-134, 2008.

[2] R. Gajapathy, K. Velusamy, and P. Selvaraj, "CFD investigation of effect of helical wire-wrap parameters on the thermal hydraulic performance of 217 fuel pin bundle," Annals of Nuclear Energy, vol. 77, pp. 498-513, 2015.

[3] L. Kefeng, C. Liuli, and Y. Chenchong, "Experimental investigation on resistance characteristics of wire-wrapped fuel assembly in Lead-Bismuth Eutectic," Nuclear Power Engineering, vol. 36, pp. 27-31, 2015.
[4] H. Kamide, K. Hayashi, and S. Toda, “An experimental study of inter-subassembly heat transfer during natural circulation decay heat removal in fast breeder reactors," Nuclear Engineering and Design, vol. 183, pp. 97-106, 1998.

[5] M. M. Sarafraz and M. Arjomandi, "Demonstration of plausible application of gallium nano-suspension in microchannel solar thermal receiver: experimental assessment of thermohydraulic performance of microchannel," International Communications in Heat and Mass Transfer, vol. 94, pp. 39-46, 2018.

[6] M. M. Sarafraz, H. Arya, and M. Arjomandi, "Thermal and hydraulic analysis of a rectangular microchannel with gallium-copper oxide nano-suspension[J]," Journal of Molecular Liquids, vol. 263, pp. 382-389, 2018.

[7] S. Hoseinzadeh, S. M. T. Otaghsara, M. H. Z. Khatir, and P. S. Heyns, "Numerical investigation of thermal pulsating alumina/water nanofluid flow over three different cross-sectional channel[J]," International Journal of Numerical Methods for Heat and Fluid Flow, vol. 30, pp. 3721-3735, 2019, ahead-ofprint(ahead-of-print).

[8] M. H. Chun and K. W. Seo, "An experimental study and assessment of existing friction factor correlations for wirewrapped fuel assemblies," Annals of Nuclear Energy, vol. 28, no. 17 , pp. 1683-1695, 2001.

[9] E. Bubelis and M. Schikorr, "Review and proposal for best fit of wire-wrapped fuel bundle friction factor and pressure drop predictions using various existing correlations," Nuclear Engineering and Design, vol. 238, no. 12, pp. 3299-3320, 2008.

[10] S. K. Cheng and N. E. Todreas, Constitutive correlations for wire-wrapped subchannel analysis under forced and mixed convection conditions. Part 1.[LMFBR], Massachusetts Inst. of Tech., Cambridge (USA), 1984.

[11] S. K. Chang, D. J. Euh, H. S. Choi, H. Kim, S. R. Choi, and H. Y. Lee, "Flow distribution and pressure loss in subchannels of a wire-wrapped 37-pin rod bundle for a sodium-cooled fast reactor," Nuclear Engineering and Technology, vol. 48, no. 2, pp. 376-385, 2016.

[12] S.-K. Chang, D.-J. Euh, S. Kim et al., "Experimental study ofthe flowcharacteristics in an SFR type 61-pin rod bundle using isokinetic sampling method," Annals of Nuclear Energy, vol. 106, pp. 160-169, 2017.

[13] S. M. A. Alavi, M. R. Safaei, O. Mahian et al., "A hybrid finiteelement/finite-difference scheme for solving the 3-D energy equation in transient nonisothermal fluid flow over a staggered tube bank[J]," Numerical Heat Transfer Part B Fundamentals, vol. 68, pp. 169-183, 2015.

[14] C. Daoxi, Q. Xiaoguang, and D. Kaiwen, "Numberical analysis of hydraulic characteristic of fast reactor wire-wrappered rod bundle assembly under low Reynolds number," Atomic Energy Science and Technology, vol. 53, pp. 1424-1432, 2020.

[15] R. Gajapathy, K. Velusamy, P. Selvaraj, P. Chellapandi, and S. C. Chetal, "CFD investigation of helical wire-wrapped 7pin fuel bundle and the challenges in modeling full scale 217 pin bundle," Nuclear Engineering and Design, vol. 237, pp. 2332-2342, 2007.

[16] F. Xukai, P. Tianji, and F. Dajun, "Study on periodicity of cross flow in gaps of a lead-based fast reactor fuel assembly," Nuclear Physics Review, vol. 35, pp. 216-223, 2018.

[17] R. Gajapathy, K. Velusamy, and P. Selvaraj, “A comparative CFD investigation of helical wire-wrapped 7,19 and 37 fuel pin bundles and its extendibility to 217 pin bundle," Nuclear Engineering and Design, vol. 239, no. 11, pp. 2279-2292, 2009. 
[18] R. Gajapathy, "CFD investigations of helical wire-wrap fuel pin bundle and its comparison with straight wire bundle," Progress in Nuclear Energy, vol. 89, pp. 57-68, 2016.

[19] U. Parthasarathy, T. Sundararajan, C. Balaji, and K. Velusamy, "Decay heat removal in pool type fast reactor using passive systems," Nuclear Engineering and Design, vol. 250, pp. 480499, 2012.

[20] J. K. Fink and L. Leibowitz, Thermodynamic and Transport Properties of Sodium Liquid and Vapor, Office of Scientific \& Technical Information Technical Reports, 1995. 